\title{
Subject Index to Volume 38
}

Abstracts

European Society for Pediatric Research, 1995 meeting, 423

Latinoamerican Society of Pediatric Endocrinology, 1994 meeting, 621

Acylcarnitine, profiling, diagnostic indicator for inborn errors of metabolism, automated electrospray tandem mass spectrometry role, 324

ADD, see Attention deficit disorder

Adenosine, endogenous, role, reducing ischemic brain injury, neonatal rats, 306

Adenoviruses, replication-deficient, gene transfer and, fetal sheep, 844

Adolescent

bone and liver alkaline phosphatase and osteocalcin, reference values for, 955

cytokine production pattern after remission of asthma, after stimulation with Dermatophagoides farinae, 187

growth hormone effects on ovarian function, 763

insulin-dependent diabetes mellitus and, growth and final height, 607

ovarian function, endocrine and ultrasound parameters, menstrual irregularities and, 974

uremia, dialysis and, insulin secretion and growth failure, 379

Airway, upper, contribution to respiratory response during seizures, piglets, 61

Airway obstruction, tidal breathing patterns and, infants, 218

Alanine, transport and utilization, during postnatal development, rat, 81

Aldosterone, effects water and electrolyte metabolism in colonic mucosal epithelium, preterm rats, 356

Alkaline phosphatase, bone and liver, osteocalcin levels and, children and adolescents, 955

All-trans-retinoic acid treatment, hemodynamic function and heart development effects, 342

American Pediatric Society, presidential address, 1995 meeting, 819

Amino acids

nitrogen balance changes in, after overnight fasting, perinatal and adult rats, 1018

transport and utilization, during postnatal development, rat, 81

Anaphylactic reaction, bee sting, allergic children, plasma catecholamine levels, 998

4-Androstenedione, $17 \alpha$-hydroxyprogesterone and testosterone, analysis by isotope dilution/gas chromatography-mass spectrometry, children, 76

Anemia

aplastic, bone marrow fibroblast exhibition of reduced IL-6 production, children with, 716 chronic fetal, atrial natriuretic peptide role in renal adaptation to, 722

Angiotensin II, receptor levels, renin-angiotensin system, fetal sheep, 896

Antigen serotyping, $\mathrm{M}$ and $\mathrm{T}$, field inversion gel electrophoresis and, epidemiology of group A Streptococcus infections, comparison study, 988

Apnea

laryngeal chemoreflex-induced, $\beta$-endorphin involvement, piglets, 205

obstructive, hypoxemia and, submental and diaphragmatic muscle activity during, preterm infants, 298

Arachidonic acid, docosahexaenoic acid and, distribution in liver, infants, 361

Arginine metabolism, nitric oxide synthesis and, persistent pulmonary hypertension of newborn, 17

Arterial blood gas, values in fetal lamb, 528

Arthritis, juvenile rheumatoid, pain levels, descriptive study, 194

Asphyxia, effects, pharmacokinetics of ceftazidime, term infants, 808

Aspirin, versus indomethacin treatment, patent ductus arteriosus, preterm infants with RDS, 886

Asthma, remission of, cytokine production pattern, after stimulation with Dermatophagoides farinae, adolescents, 187

Atopic disease, IgAl-cleaving bacteria levels, in pharynx of infants with, 182

Attention deficit disorder, catecholamine response after glucose ingestion, 539

Award, 1995 John Howland, acceptance by Floyd W. Denny, Jr., 1036

Bacteria, IgA1-cleaving, levels in pharynx of infants with atopic disease, infants, 182

Bee sting, anaphylactic reaction to, allergic children, plasma catecholamine levels, 998

Behavior, problem, long-term study of stability and change, 612

Behavioral effects, catecholamine response after glucose ingestion, children with ADD, 539

Behavioral predictors, immune function and starting school, 1009

Betamethasone, cardiovascular and metabolic effects, preterm sheep born by cesarean section, 709

Bile acids, synthesis, liver and, 156

Bile pigment metabolism, bilirubin esterification and, developing chick, 349

Biliary atresia, extrahepatic, serum hyaluronic acid and procollagen propeptide levels as prognostic tool, 568
Bilirubin

encephalopathy, visual evoked potential abnormalities and, sulfadimethoxine treatment, rats, 258

esterification, bile pigment composition and, developing chick, 349

excessive levels, long-term neurologic deficits and, 949

Blood coagulation, hyperinsulinemia effects on development of, fetus, 169

Blood flow

cerebral

norepinephrine responses, nitric oxide and $\beta$-adrenergic effects on, fetus and neonate, 237

oxygenation and, indomethacin effects, normal and ventilated fetal lamb, 243

dilatation and, neonatal intestine, 783

uterine, embryo development, diabetes during pregnancy effects, fetal rat, 598

Body composition, fat-free mass measurement, infants, 411

Bone, alkaline phosphatase, reference values, healthy children and adolescents, 955

Bone marrow, fibroblast, reduced IL- 6 production and, children with aplastic anemia, 716

$\mathrm{BPD}$, see Bronchopulmonary dysplasia

Brain

damage/injury

dizocilipine maleate and L-NAME comparison, effects on lesion size and hyperemia, 644

hypoxic-ischemic, caffeine use during pregnancy effects, neonate, 312

hypoxic-ischemic, status epilepticus effects on, postnatal rats, 251

ischemic, reduction by endogenous adenosine, neonatal rats, 306

edema, short- vs. long-term hypoxia-ischemia effects, MRI analysis, neonatal rat, 113

functional development after intrauterine hypoxia-ischemia, cerebellar granule cell analysis and, 107

phenylalanine concentrations in, patients with phenylketonuria, magnetic resonance spectroscopy measurement, 657

temperature, energy utilization rate, magnetic resonance spectroscopy analysis of relationship, 919

Breathing, periodic, temporal structure changes with postnatal development, preterm infants, 533

Breathing patterns, tidal, assessment for monitoring of bronchial obstruction, infants, 218

Bronchial obstruction, tidal breathing pattern assessment for monitoring of, infants, 218

Bronchopulmonary dysplasia, furosemide effects on airway relaxation, infants, 280 
SUBJECT INDEX TO VOLUME 38

Bypass bridge, effects on oxygenation and hemodynamics, during veno-arterial ECMO, neonates, 124

$\mathrm{Ca}^{2+}$, levels in fetal heart with retinoic acid-induced malformations, rats, 892

Caffeine, use during pregnancy, hypoxic-ischemic brain damage, neonate, 312

Cardiorespiratory arousal, submental and diaphragmatic muscle activity during, preterm infants, 298

Cardiovascular responses, betamethasone therapy, preterm sheep born by cesarean section, 709

Catecholamine, plasma levels, after bee sting, allergic children, 998

CD8 lymphocytes, activation marker expression, HIV-1-infected children, 390

Ceftazidime, pharmacokinetics, asphyxia effects on, term infants, 808

Central nervous system, hypoxia-ischemia, quisqualate effects on nitric oxide synthase neurons, role, reducing damage after, neonatal rat, 912

Cerebellar granule cells, brain functional development after intrauterine hypoxia-ischemia and, 107

Cerebral artery, occlusion, L-NAME effects, reducing infarct volume during, rats, 652

Cerebral hematoma, prostanoid role, enhancing vasoconstrictions to leukotriene $\mathrm{C}_{4}$. piglets, 119

Cerebral response, maternal cocaine injection, fetal sheep, 943

Cerebral vasculature, immaturity of, intracranial hemorrhage and, neonates, 493

Chemoreceptor, responsiveness, ketamine, morphine, pentobarbital, and propranolol effects on, fetal sheep, 938

Chemotherapy, liposome role drug delivery, 7

Child Behavior Checklist, problem behavior and, long-term study of stability and change, 612

Children

HIV-1-infected, CD8 lymphocyte activation marker expression in, 390

sickle cell disease, 6-keto-prostaglandin $\mathrm{F}_{10}$ and thromboxane plasma levels, 95

Chronic lung disease, see Lung disease, chronic

Coagulation, hyperinsulinemia effects on development of, fetus, 169

Cocaine use, see under Substance abuse

Cognitive effects, catecholamine response after glucose ingestion, children with ADD, 539

Contractile response, maturation of, effects, pulmonary vascular reactivity, newborn pigs, 25

Corticosteroid therapy, acute lymphoblastic leukemia, obesity after treatment, 86

Cortisol, insulin and, effects on surfactant protein mRNA levels, 513

Cranial irradiation, acute lymphoblastic leukemia, obesity after treatment, 86

Crying, after heel prick, sucrose effects on reducing, preterm infants, 332

Cyanosis, congenital heart disease and, sympathetic activity regulation, 55

Cysteamine therapy, intravenous, nephropathic cystinosis and, 579
Cystic fibrosis, airway epithelial cells, high molecular weight glycoconjugates and, 42

Cytokines, intratumoral production, hematopoiesis and, children with hepatoblastoma, 555

Cytomegalovirus infection, effects, endothelial cell and fibroblast growth factor production, 1003

Cytopenias, immune-mediated, neonates, 993

Denny, Floyd W., Jr., John Howland Award, 1032

Dermatophagoides farinae, cytokine production pattern during remission of asthma, after stimulation with, adolescents, 187

Development, see also Growth

dopaminerigc inhibitory mechanism operation during, puberty, 91

postnatal

age-related susceptibility to carbon tetrachloride hepatotoxicity, 140

alanine transport and utilization during, rat, 81

quotient, long-chain polyunsaturated fatty acids effects on, 4-month-old infants, 262

receptor-mediated endocytosis and, 835

Dexamethasone, surfactant protein gene expression effects, streptozotocin-induced rat lungs, 870

Diabetes mellitus

insulin-dependent, growth and final height, children, 607

during pregnancy, uterine blood flow and embryo development, 598

Diaphragm, muscle activity during obstructive apnea and cardiorespiratory arousal, preterm infants, 298

Diarrhea, rotavirus, jejunal mucosal recovery and, oral TGF- $\alpha$ effects on, piglets, 173

Dilation, flow-induced, neonatal intestine, 783

Dioxin, polychlorinated biphenyl, immunologic effects after exposure, 404

Docosahexaenoic acid, arachidonic acid and, distribution in liver, infants, 361

Dopaminergic inhibitory mechanism, operation during development, puberty, 91

Ductus arteriosus, patent, aspirin versus indomethacin treatment, infants with RDS, 886

ECMO, see Oxygenation, extracorporeal membrane

Edema, brain, hypoxia-ischemia, short- $v s$. long-term exposure, effects, brain edema, MRI analysis, neonatal rat, 113

EGF, see Epidermal growth factor

Electrophoresis, field inversion gel, antigen serotyping and, epidemiology of group A Streptococcus infections, 988

End-expiratory pressure, positive, during $\mathrm{KL}_{4}$ surfactant instillation, role, enhancing pulmonary distribution, RDS and, 211

Endocrine parameters, ovaries, adolescents with menstrual irregularities and, 974

Endocytosis, receptor-mediated, 835

$\beta$-Endorphin, laryngeal chemoreflex and, piglets, 205

Endothelial cells, growth factor production, cytomegalovirus infection effects, 1003
Endothelium, smooth muscle cell contractility effects, pulmonary vascular reactivity, newborn pigs, 25

Energy utilization rate, brain temperature and, magnetic resonance spectroscopy analysis of relationship, 919

Enteral nutrition, intestinal motor function and, preterm infants, 133

Epidermal growth factor, TGF- $\alpha$, and EGF-receptor immunolocalization, lung injury and, 851

Erythropoietin, recombinant human, effects on hemoglobin, preterm infants, 729

European Society for Pediatric Research, abstracts for 1995 meeting, 423

Expiratory flow, forced, rapid thoracoabdominal compression measurements, neonatal lambs, 664

Fat-free mass, measurement in infants, 411

Fc receptor, IgG transport by placental trophoblast, 1

Fetus

$\beta$-adrenergic and nitric oxide effects on norepinephrine, cerebral blood flow and, 237

angiotensin II receptor levels, renin-angiotensin system, sheep, 896

chemoreceptor responsiveness after hypoxemia, ketamine, morphine, pentobarbital, and propranolol effects on, sheep, 938

chronic anemia and, atrial natriuretic peptide role in renal adaptation to, 722

chronic hyperglycemic, glucose suppression of insulin secretion, 754

cocaine-exposed, cerebral response to maternal injection, sheep, 943

development, maternal diabetes and, 598

gene transfer and replication-deficient adenoviruses, sheep, 844

glycogen storage disease type II, prenatal diagnosis, 103

HLA class II-deficient combined immunodeficiency, genetic markers and prenatal diagnosis, 812

hyperinsulinemia effects on development of coagulation, 169

hypoxia-ischemia

brain functional development after, cerebellar granule cell analysis, 107

short- $v s$. long-term exposure, effects, brain edema, MRI analysis, rat, 113

nitric oxide effects on vascular tone and limb reduction, rats, 905

vasopressin effects on lung liquid volume, sheep, 30

Fibroblasts, growth factor production, cytomegalovirus infection effects, 1003

Fragile $\mathrm{X}$ syndrome

reduced cyclic AMP production, cytogenetic and molecular correlation, 639

repeat amplification mechanism and timing, 629

Furosemide, airway relaxation effects, bronchopulmonary dysplasia, 280

Ganglion, nodose, int-2 gene influence on development of, 485

Gene transfer, adenoviral vector for, fetal sheep, 
Genetic studies

fragile $X$ syndrome, repeat amplification mechanism and timing, 629

glycogen storage disease type II, prenatal diagnosis, 103

growth hormone deficiency type IB, phenotypic characteristics, 962

heart disease, effect of weaning on serum lipoprotein concentration, 522

HLA class II-deficient combined immunodeficiency, genetic markers and prenatal diagnosis, 812

Genotypes, hepatitis $\mathrm{C}$ virus, in serum and liver, children with chronic hepatitis $\mathrm{C}, 618$

GH, see Growth hormone

Glottal patency, during seizures, piglets, 932

Glucocorticoid receptor, expression by ligand, fetal rat lung cells, 506

Glucocorticoid stimulation, sympathetic innervation and, heart development effects, 479

Glucocorticoids

mineralocorticoids, effects on mRNA expression colon ion transporters, infant rats, 164

role, growth delay after liver transplantation, 366

Glucose

catecholamine response after ingestion of, children with $\mathrm{ADD}, 539$

suppression, insulin secretion, chronic hyperglycemic fetal sheep, 754

Glycine metabolism, pathways in hepatocytes, fetal sheep, 775

Glycogen storage disease, type II, prenatal diagnosis, 103

Growth, see also Development

birth to final height, small-for-gestational age infants, 733

catch-up, at 2 years of age, small-for gestational age infants, 267

delay, after liver transplantation, underlying mechanisms, 366

final height and, children with insulin-dependent diabetes mellitus, 607

quality, fat-free mass measurement and, infants, 411

receptor-mediated endocytosis and, 835

retardation, intrauterine, catch-up growth at 2 years of age, 267

slow, vitamin $\mathrm{B}_{6}$ levels association with, breast-fed infants, 740

Growth hormone

deficiency

isolated central hypothyroidism and, 967

type IB, phenotypic characteristics and genetic studies, 962

effects on ovarian function, puberty and, 763

secretion, long-term alterations in, dietary protein restriction effects, rats, 747

therapy, adipocyte mRNA levels in, GH deficiency and Prader-Willi syndrome patients, 418

Heart

development, hemodynamic function and, All-trans-retinoic acid treatment effects on, 342

ischemic contracture, mechanical and metabolic characterization, 228 malformations, retinoic acid-induced, $\mathrm{Ca}^{2+}$ levels, rats, 892

reducing equivalent shuttles in developing myocardium, neonate, 221

Heart disease

congenital

pulmonary vasculature visualization by intravascular ultrasound, infants and children, 36

retinoic acid-induced, rats, 892

sympathetic activity regulation, 55

serum lipoprotein concentration and, effect of weaning on, 522

Heel lancing, sucrose effects reducing pain, preterm infants, 332

Height, final, growth and

children with insulin-dependent diabetes mellitus, 607

small-for-gestational age infants, 733

Hematopoiesis, intratumoral cytokine production and, children with hepatoblastoma, 555

Hemoglobin, recombinant human erythropoietin effects on, preterm infants, 729

Hemorrhage, intracranial, cerebral vasculature immaturity and, neonates, 493

Hepatitis $\mathrm{C}$, chronic, genotypes in serum and liver, children, 618

Hepatoblastoma, hematopoiesis and intratumoral cytokine production, children, 555

Hepatocyte, fetal, serine and glycine metabolism pathways, fetal sheep, 775

Hepatotoxicity, age-related susceptibility to carbon tetrachloride, 140

HIV, see Human immunodeficiency virus

HLA class II-deficient combined immunodeficiency, genetic markers and prenatal diagnosis, 812

Hormonal regulation, testosterone secretion and, cells isolated from testes, infants and children, 592

Hormone therapy, cardiovascular and metabolic effects, preterm sheep born by cesarean section, 709

Human immunodeficiency virus-1

CD8 lymphocyte activation marker expression in, children, 390

maternal-child transmission, identification of factors involved in, 384

tat, neuroblast adhesion to, infants and children, 792

Hyaluronic acid, serum, procollagen propeptide levels, role, prognostic indicator of extrahepatic biliary atresia, 568

$17 \alpha$-Hydroxyprogesterone, 4-androstenedione and testosterone, analysis by isotope dilution/gas chromatography-mass spectrometry, children, 76

Hyperemia, cerebral, dizocilipine maleate effects, 644

Hyperglycemia, chronic, glucose suppression of insulin secretion, fetal sheep, 754

Hyperinsulinemia, blood coagulation development effects, fetus, 169

Hyperoxia

hydroxyl radical mediation and, preterm infants, 286

propylthiouracil effects on lung injury after, rats, 292

pulmonary neutrophil comparison, adult and neonatal rats after, 857
TGF- $\alpha$ secretion by alveolar macrophages, postnatal rabbits after, 49

Hypertension

persistent pulmonary, arginine metabolism and nitric oxide synthesis, neonate, 17

pulmonary, acute respiratory failure, inhaled nitric oxide $v s$. inhaled prostacyclin, piglets, 198

Hypoplasia, lung, short-term obstruction of trachea role in reversing, fetal sheep, 690

Hypothyroidism, isolated central, short stature and, 967

Hypoxemia

chemoreceptor responsiveness and, ketamine, morphine, pentobarbital, and propranolol effects on, fetal sheep, 938

theophylline effects on metabolic and ventilatory response to, during quiet sleep, piglets, 926

Hypoxia, antenatal vitamin $\mathrm{E}$ effects on changes induced by, neonatal rats, 685

Hypoxia-ischemia

brain damage, status epilepticus effects, postnatal rats, 251

brain damage and, caffeine use during pregnancy effects, neonate, 312

intrauterine, brain functional development effects, cerebellar granule cell analysis and, 107

quisqualate destruction of nitric oxide synthase neurons, role, reducing damage after, neonatal rat, 912

short- $v s$. long-term exposure, effects, brain edema, MRI analysis, neonatal rat, 113

IGF, see Insulin-like growth factor

IgG, see Immunoglobulin $\mathrm{G}$

Immune function, starting school and, adrenocortical and behavioral predictors of, 1009

Immunodeficiency, HLA class II-deficient combined, genetic markers and prenatal diagnosis, 812

Immunoglobulin A, secretory, bacteria capable of cleaving, levels in pharynx of infants with atopic disease, 182

Immunoglobulin $\mathrm{G}$, transport by placental trophoblast,

Immunolocalization, TGF- $\alpha, \mathrm{EGF}$, and EGF-receptor, lung injury and, 851

Immunologic effects, dioxin and polychlorinated biphenyl exposure, 404

Immunosuppressive, HLA-derived peptides as, 275

Inborn errors of metabolism, acylcarnitine and amino acid profiling as diagnostic tool, automated electrospray tandem mass spectrometry role, 324

Indomethacin

cerebral blood flow and oxygenation effects, normal and ventilated fetal lamb, 243

versus aspirin treatment, patent ductus arteriosus, preterm infants with RDS, 886

Infant

absorption of supplemental iron, 373

asphyxia effects on ceftazidime pharmacokinetics, 808

atopic disease, IgA1-cleaving bacteria levels, in pharynx of infants with, 182 
SUBJECT INDEX TO VOLUME 38

breast-fed, vitamin $\mathrm{B}_{6}$ levels association with slow growth in, 740

bronchopulmonary dysplasia, furosemide, airway relaxation effects, 280

development quotient, long-chain polyunsaturated fatty acids effects on, 4-month-old, 262

excessive bilirubin levels, long-term neurologic deficits and, 949

fat-free mass measurement, 411

HIV- 1 and, maternal child vertical transmission, identification of factors involved in, 384

HIV-1 tat, neuroblast adhesion to, 792

lung function testing, tidal breathing pattern role, 218

myocardial contracture and ischemia, mechanical and metabolic characterization, 228

persistent pulmonary hypertension, arginine metabolism and nitric oxide synthesis, 17

preterm

betamethasone treatment, cardiovascular and metabolic effects, sheep born by cesarean section, 709

chronic lung disease, IL-8 effects, 11

chronic lung disease oxidative stress role in development of, 286

enteral nutrition, intestinal motor function and, 133

intrauterine vitamin $\mathrm{B}_{12}$ uptake, 585

obstructive apnea and cardiorespiratory arousal, after hypoxemia, submental and diaphragmatic muscle activity during, 298

periodic breathing, temporal structure changes with postnatal development, 533

RDS, aspirin versus indomethacin treatment of patent ductus arteriosus, 886

recombinant human erythropoietin effects on hemoglobin, 729

sucrose effects reducing pain during heel lancing, 332

pulmonary vascular disease, intravascular ultrasound visualization feasibility, 36

small-for-gestational age

catch-up growth at 2 years of age, 267

full-term, growth from birth to final height, 733

TGF- $\alpha$ secretion by alveolar macrophages, postnatal rabbits after hyperoxia, 49

Inflammatory response, meningeal, group B Insulin Streptococcus, neonatal piglet, 981

cortisol and, effects on surfactant protein mRNA levels, 513

secretion

glucose suppression of, chronic

hyperglycemic fetal sheep, 754

growth failure and, adolescents with uremia on dialysis, 379

long-term alterations in, dietary protein restriction effects, rats, 747

Insulin-like growth factor, binding protein-3 and IGF-I, levels during adolescence and childhood, 149

Int-2 gene, nodose ganglion development influence, 485

Interleukin-6, bone marrow fibroblast effects, children with aplastic anemia, 716
Interleukin-8, neutrophil chemoattractants, effects, chronic lung disease of prematurity, 11

Interleukin-1, receptor antagonist, effects on hemodynamic manifestations of group B streptococcal sepsis, 704

Intestine, neonatal, flow-induced dilation and 783

Intracranial pressure, thoracoabdominal compression effects on, neonatal lambs, 664

Ion transporters, colon, mRNA expression in, glucocorticoids and mineralocorticoids effects on, infant rats, 164

Iron, supplemental, absorption of, early infancy, 373

Ischemia

brain injury and, endogenous adenosine role in reducing, neonatal rats, 306

myocardial contracture and, mechanical and metabolic characterization, 228

IUGR, see Growth retardation, intrauterine

Jaundice, visual evoked potential abnormalities and, sulfadimethoxine treatment effects on, rats, 258

Jejunal mucosa, recovery, rotavirus diarrhea and, oral TGF- $\alpha$ effects on, piglets, 173

John Howland Award presentation, to Floyd W. Denny, Jr., 1032

Jugular venous occlusion, near-infrared spectroscopy and, role, measuring cerebra venous oxyhemoglobin saturation, 319

Kawasaki disease, effects on migration of umbilical cord endothelial cells, 501

Ketamine, chemoreceptor responsiveness effects, fetal sheep, 938

Kidney

disease, end-state, insulin secretion and growth failure, adolescents with uremia on dialysis, 379

function, angiotensin II receptor levels and, fetal sheep, 896

Laryngeal chemoreflex, $\beta$-endorphin involvement in apnea induced by, piglets, 205

Latinoamerican Society of Pediatric Endocrinology, abstracts of 1994 meeting, 621

Leukemia

acute lymphoblastic, obesity after treatment, 86 childhood acute, late effects and side effects, long-term survivors, follow-up study, 802

Leukotriene $\mathrm{B}_{4}$, effects, chronic lung disease of prematurity, 11

Leydig cell activation, hormonal regulation of early postnatal phase, 592

Limb reduction, nitric oxide-induced, fetal rats, 905

Lipid, exogenous, placental transfer and, pregnant rabbit, 1026

Lipoprotein concentration, serum, effect of weaning on, heart disease and, 522

Liposome, role, drug delivery for chemotherapy, 7

Liver

alanine transport and utilization during postnatal development, rat, 81

alkaline phosphatase, reference values, healthy children and adolescents, 955 arachidonic acid and docosahexaenoic acid distribution in, infants, 361

bile acid and bilirubin synthetization, 150

bilirubin esterification and bile pigment metabolism, developing chick, 349

disease, growth delay after transplantation, underlying mechanisms, 366

hepatitis $\mathrm{C}$, genotypes in serum and liver, children, 618

nitrogen balance changes in amino acids and hormone levels, after overnight fasting, perinatal and adult rats, 1018

transplantation, growth delay after, underlying mechanisms, 366

Lung

disease, chronic

IL-8, leukotriene $\mathrm{B}_{4}$, and platelet-activating factor effects, preterm infant, 11

oxidative stress role in development of, ventilated preterm infants, 286

function, dexamethasone and exogenous surfactant therapy effects on, 676

function tests, tidal breathing patterns and, infants, 218

injury

propylthiouracil effects, after hyperoxia, rats, 292

TGF- $\alpha$, EGF, and EGF-receptor immunolocalization, 851

liquid volume, vasopressin effects, fetal sheep, 30

streptozotocin-induced, dexamethasone effects on surfactant protein gene expression, rats, 870

surfactant deficiency, intratracheal pulmonary versus mechanical ventilation, rabbits, 878

surfactant protein B developmental and glucocorticoid regulation, fetus, 668

Lymphoproliferative disease, $\mathrm{X}$-linked, review study, 471

Macrophages, alveolar, TGF- $\alpha$ secretion by, postnatal rabbits after hyperoxia, 49

Magnetic resonance imaging, hypoxia-ischemia, short- $v s$. long-term exposure, effects, brain edema, neonatal rat, 113

Magnetic resonance spectroscopy, ${ }^{31} \mathrm{P}$ and ${ }^{1} \mathrm{H}$, brain temperature and energy utilization rate relationship, 919

Mammary tumor virus, int-2 gene and, nodose ganglion development influence, 485

Maternal cocaine injection, cerebral response to, fetal sheep, 943

Maternal-child interactions, HIV-1 transmission

CD8 lymphocyte activation marker expression in, 390

identification of factors involved in, 384

Mental retardation, fragile $\mathrm{X}$ syndrome, repeat amplification mechanism and timing, 629

Metabolic response, hypoxemia, theophylline effects, during quiet sleep, piglets, 926

Mineralocorticoids, glucocorticoids and, effects on mRNA expression colon ion transporters, infant rats, 164

Monoclonal antibody, reshaped, protective effects, respiratory syncytial virus, rats, 543

Mononuclear phagocytic system, liposomes and, role, drug delivery for chemotherapy, 7 
Morphine, chemoreceptor responsiveness effects, fetal sheep, 938

Motor activity, intestinal, postnatal change in, 133

Myocardium, developing, reducing equivalent shuttles in, newborn heart, 221

L-NAME, see $N^{\mathrm{G}}$-nitro-L-arginine methyl ester NDMA, see $N$-methyl-D-aspartic acid

Neonate

$\beta$-adrenergic and nitric oxide effects on norepinephrine, cerebral blood flow and, 237

hypoxia-ischemia

brain damage, caffeine use during pregnancy effects, 312

quisqualate destruction of nitric oxide synthase neurons, role, reducing damage after, rats, 912

intracranial pressure, thoracoabdominal compression effects on, neonatal lambs, 664

myocardial contracture and ischemia, mechanical and metabolic characterization, 228

periodic breathing, temporal structure changes with postnatal development, 533

persistent pulmonary hypertension, arginine metabolism and nitric oxide synthesis, 17

Nephropathic cytinosis, intravenous cysteamine therapy effects, 579

Neuroblast adhesion, HIV-1 tat, infants and children, 792

Neurodevelopment quotient, long-chain polyunsaturated fatty acids effects on, 4-month-old infants, 262

Neurologic deficits, excessive bilirubin levels and, neonates, 949

Neuronal injury, NMDA receptor and nitric oxide comparison, lesion size and cerebral hyperemia effects, 644

Neutropenia, immune-mediated, neonates, 993

Neutrophil

chemoattractant, IL-8, leukotriene $\mathrm{B}_{4}$, and platelet-activating factor, effects, chronic lung disease of prematurity, 11

pulmonary, comparison study, adult and neonatal rats after hyperoxia, 857

$N^{G}$-nitro-L-arginine methyl ester

dizocilipine maleate and, effects on lesion size and hyperemia, comparison study, 644

effects, reducing infarct volume during cerebral artery occlusion, rats, 652

Nitric oxide

$\beta$-adrenergic and, effects on norepinephrine, cerebral blood flow and, fetus and neonate, 237

inhaled, $v s$. inhaled prostacyclin, pulmonary hypertension with acute respiratory failure, piglets, 198

vascular tone and limb reduction effects, during fetal development, rats, 905

Nitric oxide synthase

arginine metabolism and, persistent pulmonary hypertension of newborn, 17

neurons, quisqualate destruction of, role, reducing damage after hypoxia-ischemia, neonatal rat, 912 perinatal inhibition, effects, neonatal growth and induction of hypertorphic pyloric stenosis, rats, 768

Norepinephrine, cerebral blood flow and, nitric oxide and $\beta$-adrenergic effects on, fetus and neonate, 237

Nutritional status, fat-free mass measurement and, infants, 411

Obesity, after acute lymphoblastic leukemia treatment, 86

Ornithine decarboxylase, tyrosine kinase activity and, juvenile polyps, 574

Osteocalcin, reference values, healthy children and adolescents, 955

Ovarian function, growth hormone effects on, puberty and, 763

Ovarian parameters, endocrine and ultrasound, adolescents with menstrual irregularities and, 974

Oxidative stress, role, development of chronic lung disease, preterm infants, 286

\section{Oxygenation}

cerebral blood flow and, indomethacin effects, normal and ventilated fetal lamb, 243

extracorporeal membrane, veno-arterial, bypass bridge opening effects on oxygenation and hemodynamics, neonates, 124

Oxyhemoglobin saturation, cerebral venous, near-infrared spectroscopy and jugular venous occlusion measurement, 319

Pain assessment, juvenile rheumatoid arthritis, descriptive study, 194

Pediatrics, language of, presidential address, 819

Pentobarbital, chemoreceptor responsiveness effects, fetal sheep, 938

Phenylketonuria, phenylalanine concentrations in brain, magnetic resonance spectroscopy measurement, 657

Placental trophoblast, IgG transport mechanism, Platelet-activating factor, effects, chronic lung disease of prematurity, 11

Polychlorinated biphenyl, dioxins and, immunologic effects after exposure, 404

Polyps, juvenile, ornithine decarboxylase and tyrosine kinase activity, 574

Polyunsaturated fatty acids, long-chain, effects on development quotient, 4-month-old infants, 262

Prader-Willi syndrome, growth hormone therapy effects on adipocyte mRNA levels in, 418

Pregnancy

caffeine use during, effects on hypoxic ischemia, neonate, 312

diabetes during, uterine blood flow and embryo development, rats, 598

Presidential address

American Pediatric Society, 1995: Disease, War, and Biology: Languages for Medicine-and Pediatrics, 819

Society for Pediatric Research, 1995: Marrying our Medicine to Biology, 830

Propranolol, chemoreceptor responsiveness effects, fetal sheep, 938

Propylthiouracil, lung injury effects, after hyperoxia, rats, 292

Prostacyclin, inhaled, vs. inhaled nitric oxide, pulmonary hypertension with acute respiratory failure, piglets, 198
Prostanoid, role, enhancing vasoconstrictions to leukotriene $\mathrm{C}_{4}$, cerebral hematoma and piglets, 119

Protein restriction, dietary, long-term alterations in growth hormone and insulin secretion after temporary, rats, 747

Puberty

development during, effects on IGF binding protein-3 and IGF-I levels, 149

growth hormone effects on ovarian function 763

Pulmonary neutrophils, comparison study, adult and neonatal rats after hyperoxia, 857

Pulmonary stenosis, sympathetic activity regulation, 55

Pulmonary surfactant, CTP:phosphocholine cytidylyltransferse by cytosolic lipids in rat type II pneumocytes during development, 864

Pulmonary vascular reactivity, contractile response maturation and endothelial modulation effects, newborn pigs, 25

Pulmonary vasculature, intravascular ultrasound feasibility, infants and children, 36

Quisqualate, nitric oxide synthase neuron destruction and, role, reducing damager after hypoxia-ischemia, neonatal rat, 912

RDS, see Respiratory distress syndrome

Renin-angiotensin system, angiotensin II receptor levels, fetal sheep, 896

Respiratory distress syndrome aspirin versus indomethacin treatment of patent ductus arteriosus, preterm infants, 886

positive end-expiratory pressure during $\mathrm{KL}_{4}$ surfactant instillation, role, enhancing pulmonary distribution, 211

Respiratory failure, acute, pulmonary hypertension with, inhaled nitric oxide $v s$ inhaled prostacyclin, piglets, 198

Respiratory response, during seizures, upper airway contribution to, piglets, 61

Respiratory support, during seizures, glottal patency and, piglets, 932

Respiratory syncytial virus, reshaped monoclonal antibody RSHZ19 protective efficacy, rats, 543

Retinoic acid, heart malformations and, $\mathrm{Ca}^{2+}$ levels, rats, 892

School, immune function and, adrenocortical and behavioral predictors of, 1009

Seizure

glottal patency during, piglets, 932

upper airway contribution to respiratory response during, piglets, 61

L-Selectin, levels in umbilical cord blood, increase with gestational age, 336

Sepsis, group B streptococcal

IL-1 receptor antagonist effects on hemodynamic manifestations of, 704

TNF- $\alpha$ antibodies effects on, neonatal rats, 551

Serine metabolism, pathways in ovine fetal hepatocytes, 775

Sickle cell disease, 6-keto-prostaglandin $F_{1 \alpha}$ and thromboxane plasma levels, 95

Sleep, quiet, theophylline effects on metabolic and ventilatory response to hypoxemia during, piglets, 926 


\section{SUBJECT INDEX TO VOLUME 38}

Small intestine, motor function, enteral nutrition and, preterm infants, 133

Smooth muscle, myosin heavy chain isoform expression, 697

Society for Pediatric Research, presidential address, 1995 meeting, 830

Spectrometry

electrospray tandem mass, role, diagnosing inborn errors of metabolism by acylcarnitine profiling, 324

isotope dilution/gas chromatography-mass, $17 \alpha$-hydroxyprogesterone, 4-androstenedione, and testosterone analysis, children, 76

Spectroscopy

magnetic resonance, phenylalanine concentrations in brain, patients with phenylketonuria, 657

near-infrared, jugular venous occlusion and, role, measuring cerebral venous oxyhemoglobin saturation, 319

Spirometry, rapid thoracoabdominal compression. method, measuring forced expiratory flow, neonatal lambs, 664

Stature, short, isolated central hypothyroidism and, 967

Status epilepticus, effects on hypoxic-ischemic brain damage, postnatal rats, 251

Stenosis, hypertrophic pyloric, perinatal nitric oxide synthase inhibition effects on retardation of, rats, 768

Streptococcus

group $\mathrm{A}$, epidemiology of infections, $\mathrm{M}$ and $\mathrm{T}$ antigen serotyping and field inversion gel electrophoresis, comparison study, 988

group B

meningeal inflammatory response, neonatal piglet, 981

sepsis, IL- 1 receptor antagonist effects on hemodynamic manifestations of, 704

TNF- $\alpha$ antibodies effects on, neonatal rats, 551

Submental muscle activity, during obstructive apnea and cardiorespiratory arousal, preterm infants, 298

Substance abuse, maternal cocaine injection, cerebral response, fetal sheep, 943

Sucrose, heel lancing pain reduction effects, preterm infants, 332

Sudden infant death syndrome, obstructive apnea and cardiorespiratory arousal and, submental and diaphragmatic muscle activity during, preterm infants, 298

Sulfadimethoxine, visual evoked potential abnormalities and jaundice, rats, 258
Sulfate transport, glycoconjugates and, cystic fibrosis airway epithelial cells, 42

\section{Surfactant}

deficiency, intratracheal pulmonary versus mechanical ventilation, rabbits, 878

$\mathrm{KL}_{4}$, end-expiratory pressure during instillation of, role, enhancing pulmonary distribution, RDS and, 211

production, glucocorticoid receptor mediation of, 506

protein, mRNA levels, insulin and cortisol effects on, 513

protein B, developmental and glucocorticoid regulation, fetal lung, 668

protein gene expression, dexamethasone effects, streptozotocin-induced rat lungs, 870

therapy, dexamethasone and exogenous, prenatal, lung function effects, 676

Sympathetic activity, regulation, congenital heart disease, 55

Sympathetic innervation, glucocorticoid stimulation, heart development effects, 479

Teniposide, liposomal formulation and, role, drug delivery for chemotherapy, 7

Testosterone

4-androstenedione and $17 \alpha$ hydroxyprogesterone, analysis by isotope dilution/gas chromatography-mass spectrometry, children, 76

secretion, growth hormone responses and, cells isolated from testes, infants and children, 592

Tetralogy of Fallot, sympathetic activity regulation, 55

TGF, see Transforming growth factor

Theophylline, metabolic and ventilatory response to hypoxemia, during quiet sleep, piglets, 926

Thrombocytopenia, immune-mediated, neonates, 993

Thrombosis, hyperinsulinemia effects on coagulation development and, fetus, 169

Thromboxane plasma levels, sickle cell disease, children, 95

TNF, see Tumor necrosis factor

Tracheal obstruction, short-term, role, reversing lung hypoplasia, fetal sheep, 690

Transforming growth factor- $\alpha$

effects on jejunal mucosal recovery in rotavirus diarrhea, piglets, 173

EGF, and EGF-receptor immunolocalization, lung injury and, 851 secretion by alveolar macrophages, postnatal rabbits after hyperoxia, 49

Transplantation, see specific types

Tumor, germ cell, tumor markers CA 125 and CA $19-9$ in cord blood during infancy and, 797

Tumor markers, CA 125 and CA 19-9, in cord blood during infancy, use in germ cell tumors, 797

Tumor necrosis factor- $\alpha$, antibodies effects on group B Streptococcus, neonatal rats, 551

Tyrosine kinase, ornithine decarboxylase activity and, juvenile polyps, 574

Ultrasound

intravascular, pulmonary vasculature visualization and, infants and children, 36

parameters, ovaries, adolescents with menstrual irregularities and, 974

Umbilical cord

blood

cytokine receptor expression, 397

polymorphonuclear leukocytes, D-myo-Inositol 1,4,5-triphosphate generation, 564

L-selectin levels, increase with gestational age, 336

tumor markers CA 125 and CA 19-9 during infancy, use in germ cell tumors, 797

endothelial cells, Kawasaki disease effect on migration of, 501

Uremia, insulin secretion and growth failure, adolescents on dialysis, 379

Vascular tone, nitric oxide effects, fetal rats, 905

Vasopressin, lung liquid volume and, fetal sheep, 30

Ventilation, intratracheal pulmonary, versus mechanical, surfactant deficiency, rabbits, 878

Ventilatory response, hypoxemia, theophylline effects, during quiet sleep, piglets, 926

Vitamin $B_{6}$, levels, association with slow growth, breast-fed infants, 740

Vitamin $B_{12}$, intrauterine uptake, preterm and full-term infants, 585

Vitamin E, antenatal, effects on hypoxia-induced changes, neonatal rats, 685

Water metabolism, colonic mucosal epithelium, aldosterone effects, preterm rats, 356

$\mathrm{X}$-linked lymphoproliferative disease, review study, 471 